Draft VERSION DECEMBER 10, 2019

Preprint typeset using $\mathrm{IATEX}_{\mathrm{E}}$ style emulateapj v. 01/23/15

\title{
FILLING THE MASS GAP: HOW KILONOVA OBSERVATIONS CAN UNVEIL THE NATURE OF THE COMPACT OBJECT MERGING WITH THE NEUTRON STAR
}

\author{
C. Barbieri ${ }^{1,3 *}$, O. S. Salafia ${ }^{2,3}$, M. Colpi ${ }^{1,3}$, G. Ghirlanda ${ }^{2,3}$, A. Perego ${ }^{3,4}$ And A. Colombo ${ }^{1}$ \\ Draft version December 10, 2019
}

\begin{abstract}
In this letter we focus on the peculiar case of a coalescing compact-object binary whose chirp mass is compatible both with a neutron star-neutron star and black hole-neutron star system, with the black hole in the $\sim 3-5 \mathrm{M}_{\odot}$ range defined as the mass gap". Some models of core-collapse supernovae predict the formation of such low-mass black holes and a recent observation seems to confirm their existence. Here we show that the nature of the companion to the neutron star can be inferred from the properties of the kilonova emission once we know the chirp mass, which is the best constrained parameter inferred from the gravitational signal in low-latency searches. In particular, we find that the kilonova in the black hole-neutron star case is far more luminous than in the neutron star-neutron star case, even when the black hole is non spinning. The difference in the kilonovae brightness arises primarily from the mass ejected during the merger. Indeed, in the considered interval of chirp masses, the mass ejection in double neutron star mergers is at its worst as the system promptly forms a black hole. Instead mass ejection for black hole-neutron star case is at its best as the neutron stars have low mass/large deformability. The kilonovae from black hole-neutron star systems can differ by two to three magnitudes. The outcome is only marginally dependent on the equation of state. The difference is above the systematics in the modeling.
\end{abstract}

Keywords: stars:neutron, stars: black holes, binaries: general, gamma-ray burst: general, gravitational waves

\section{INTRODUCTION}

During the $\mathrm{O} 1$ and $\mathrm{O} 2$ observing runs, the LIGO Scientific Collaboration and Virgo Collaboration (LVC) detected gravitational wave (GW) signals from ten coalescing stellar-mass black hole binaries $(\mathrm{BHBH})$ and a neutron star binary system (NSNS), the latter accompanied by a multi-wavelength electromagnetic (EM) counterpart (Abbott et al. 2017a,b; The LIGO Scientific Collaboration \& the Virgo Collaboration 2018). At the time of writing, as the third observing run (O3) is in progres, the LVC reported the detection of two probable black hole-neutron star (BHNS) binary merger candidates (S190814bv - The LIGO Scientific Collaboration \& the Virgo Collaboration 2019a, and S190910d - The LIGO Scientific Collaboration \& the Virgo Collaboration $2019 \mathrm{~b}$ ), plus candidates with a lower probability of being actual astrophysical events ${ }^{5}$. Before the beginning of O3, the estimated BHNS detection rate for this run was in the range $0.04-12 \mathrm{yr}^{-1}$ (Dominik et al. 2015). At the time of writing, there are no indications of observed EM counterparts associated with these candidates (Coughlin et al. 2019; for S190814bv see e.g. Srivastav 2019; Soares-Santos 2019; Klotz 2019, for S190910d see e.g.

\footnotetext{
* c.barbieri@campus.unimib.it

${ }^{1}$ Universit degli Studi di Milano-Bicocca, Dipartimento di Fisica "G. Occhialini", Piazza della Scienza 3, I-20126 Milano, Italy

2 INAF - Osservatorio Astronomico di Brera, via E. Bianchi 46, I-23807 Merate, Italy

3 INFN - Sezione di Milano-Bicocca, Piazza della Scienza 3, I-20126 Milano, Italy

${ }^{4}$ Università degli Studi di Trento, Dipartimento di Fisica, via Sommarive 14, I-38123 Trento, Italy

${ }^{5}$ We defer to the LIGO/Virgo O3 Public Alerts webpage https: //gracedb.ligo.org/superevents/public/03/for a complete list of current candidates.
}

Crisp 2019; Pereyra 2019).

On a theoretical point of view, BHNS mergers can be accompanied by an EM counterpart as in the NSNS case. This occurs when the NS is (at least partially) tidally disrupted before crossing the $\mathrm{BH}$ event horizon (Shibata \& Taniguchi 2011). Tidal disruption is favoured in binaries with low mass ratio $q=M_{1} / M_{2}$ and large NS tidal deformability $\Lambda_{\mathrm{NS}}$ (corresponding to a small NS mass and/or to a "stiff" equation of state). A high black hole spin $^{6}$, which brings the last stable circular orbit of the binary closer to the $\mathrm{BH}$ horizon, also greatly enhances the tidal disruption (Shibata \& Taniguchi 2011; Kawaguchi et al. 2016; Foucart et al. 2018; Barbieri et al. 2019b,a). The unbound NS material ("ejecta") is thought to produce kilonova emission (Lattimer \& Schramm 1974; Li \& Paczyński 1998; Metzger 2017). Moreover, Shapiro (2017); Paschalidis (2017); Ruiz et al. (2018) showed that after a BHNS merger a relativistic jet can be launched, powering a short gamma-ray burst (sGRB) (Eichler et al. 1989; Narayan et al. 1992) and GRB afterglow emission (Sari et al. 1998; D'Avanzo et al. 2018; Ghirlanda et al. 2018; Salafia et al. 2019).

The BH mass distribution observed so far in coalescing binaries is broad (The LIGO Scientific Collaboration et al. 2018), extending up to $50_{-10.2}^{+16.6} \mathrm{M}_{\odot}$, with the lightest $\mathrm{BH}$ carrying a mass $7.6_{-2.1}^{+1.3} \mathrm{M}_{\odot}$, close to the mean $\mathrm{BH}$ mass in observed Galactic X-ray binaries of $\sim 7.8 \pm 1.2 \mathrm{M}_{\odot}$ (Özel et al. 2010). Double NS systems observed so far carry masses in the interval $1.165 \mathrm{M}_{\odot}-1.590 \mathrm{M}_{\odot}$ (Zhang et al. 2019), and the NS with the highest and best estimated mass is the radio

\footnotetext{
6 We use the term "spin" to indicate the dimensionless spin parameter, $\chi_{\mathrm{BH}}=c J / G M_{\mathrm{BH}}^{2}$, where $J$ is the $\mathrm{BH}$ angular momentum.
} 
pulsar $\mathrm{J} 0740+6620$ with $M_{\mathrm{NS}}=2.14_{-0.09}^{+0.10} \mathrm{M}_{\odot}$ in a lowmass binary (Cromartie et al. 2019). Thus, observations appear to indicate a discontinuity between the observed mass distributions of NSs and stellar BHs, called mass gap", located approximately between $\approx 3 \mathrm{M}_{\odot}$ (the maximum NS mass inferred from causality arguments) and $\sim 5 \mathrm{M}_{\odot}$ (Lattimer \& Prakash 2001). However recently Thompson et al. (2019) reported the discovery of a $\mathrm{BH}$ with mass $3.3_{-0.7}^{+2.8} \mathrm{M}_{\odot}$ in a non-interacting binary system with a red giant.

The mass spectrum of compact objects depends sensitively on the mass of the carbon-oxygen core at the end of stellar evolution, on the compactness of the collapsing core at bounce and on the supernova (SN) explosion engine. Belczynski et al. (2012) and Fryer et al. (2012) showed that, in the presence of a significant amount of fallback, explosions happening over a large interval of post-bounce times lead to a continuous range in remnant masses. By contrast explosions happening predominantly within a few hundreds of ms after bounce, characterized by negligible amounts of fallback material, produce more easily the mass gap. Interestingly, at the time of writing, the LVC reported event candidates with binaries having at least one component in the mass gap (The LIGO Scientific Collaboration \& the Virgo Collaboration $2019 \mathrm{c}, \mathrm{d}$ ).

It is known that the binary chirp mass $M_{\mathrm{c}}$, a combination of the masses of the two components, is one of the best measured parameters encoded in the GW signal. It is the prime parameter used to identify in low-latency searches the nature of the binary - whether the system hosts two NSs, two stellar BHs or a BH and a NS. Interestingly, we note that if the $\mathrm{NS}$ and $\mathrm{BH}$ mass spectra join to form a continuum, i.e. there is no "mass gap" between BH and NS mass distributions (as Thompson et al. 2019 seem to indicate), there exists a range of values of the chirp mass $M_{\mathrm{c}}$ where the nature of the binary cannot be identified uniquely based on the chirp mass only (see also Mandel et al. 2015). In particular, hereafter we call "ambiguous" the chirp masses whose values are compatible with either a NSNS or a light BHNS system (see Fig. 1).

In this Letter, we aim at answering the following question: can EM observations of coalescing binaries in this "ambiguous" chirp mass interval help to disentangle their nature and narrow down the uncertainties on the existence of a mass gap? To this purpose, we study the properties of the kilonova emission of NSNS and BHNS systems which fall in this "ambiguous" chirp mass interval, using the semi-analytical model presented in Barbieri et al. (2019b,a).

\section{2. "AMBIGUOUS" CHIRP MASSES}

The binary chirp mass is defined as

$$
M_{\mathrm{c}}=\frac{\left(M_{1} M_{2}\right)^{3 / 5}}{\left(M_{1}+M_{2}\right)^{1 / 5}},
$$

where $M_{1}$ and $M_{2}$ are the masses of the two component stars (we take $M_{1} \geq M_{2}$ ). LVC public alerts follow a classification scheme to communicate probabilistic estimates of the nature of the merging system to the community. The scheme classifies as "BNS" any system with both masses $M_{1}$ and $M_{2}$ smaller than $3 \mathrm{M}_{\odot}$; as "BBH" any system with both $M_{1}$ and $M_{2}$ larger than $5 \mathrm{M}_{\odot}$; as

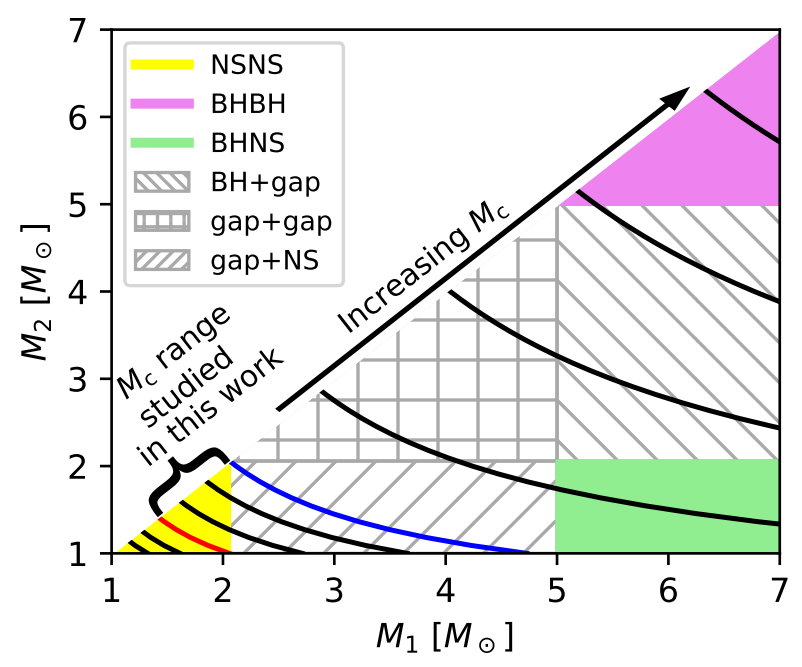

Figure 1. $M_{1}-M_{2}$ compact binaries having the same chirp mass $M_{\mathrm{c}}$. Different lines indicate different values for $M_{\mathrm{c}}$. We assume the SFHo EoS: the maximum NS mass is $M_{\mathrm{NS}}^{\max }=2.058 \mathrm{M}_{\odot}$, and $M_{\mathrm{NS}}^{\min }$ is set equal to $1 \mathrm{M}_{\odot}$. Yellow, green and violet regions of the parameter space indicate, respectively, NSNS, BHNS and $\mathrm{BHBH}$ binaries. Gray-hatched areas indicate binary configurations with at least one component in the mass gap. Red and blue lines represent $M_{\mathrm{c}, \mathrm{min}}^{\mathrm{gapNS}}$ and $M_{\mathrm{c}, \max }^{\mathrm{NSNS}}$, respectively (see text for definition).

"NSBH" any system with $M_{1}>5 \mathrm{M}_{\odot}$ and $M_{2}<3 \mathrm{M}_{\odot}$, and as "MassGap" any system with at least one component carrying a mass between 3 and $5 \mathrm{M}_{\odot}$. An additional "Terrestrial" category is defined to represent triggers that are not of astrophysical origin (i.e. false alarms).

In this work we follow a slightly different classification. We assume the SFHo equation of state (EoS), for which the maximum mass of a non-rotating NS is $M_{\mathrm{NS}}^{\max }=2.058 \mathrm{M}_{\odot}$ (Steiner et al. 2013). We also fix the minimum NS mass to $M_{\mathrm{NS}}^{\min }=1 \mathrm{M}_{\odot}(\sim 10 \%$ lower than the value found in Suwa et al. 2018). We thus classify as "NSNS" those systems with both $M_{1}$ and $M_{2}$ between 1 and $2.058 \mathrm{M}_{\odot}$ (yellow region in Fig. 1); "BHNS" those with $M_{1}>5 \mathrm{M}_{\odot}$ and $M_{2}<2.058 \mathrm{M}_{\odot}$ (green region); "BHBH" those with both masses above $5 \mathrm{M}_{\odot}$ as in the LVC classification (purple region). Considering that compact objects populating the mass gap have masses larger than $M_{\mathrm{NS}}^{\max }$, we assume these to be stellar-origin BHs. In Fig. 1 we divide the "MassGap" region in three sub-regions: "BH+gap" for those systems with a BH above $5 \mathrm{M}_{\odot}$ and a $\mathrm{BH}$ in the gap; "gap+gap" for those with two BHs in the gap; "gap+NS" for those with a BH in the gap and a NS.

Two limiting values of the chirp mass can be identified: $M_{\mathrm{c}, \text { min }}^{\text {gapNS }}=1.233 \mathrm{M}_{\odot}$ is the chirp mass corresponding to a gap $+\mathrm{NS}$ binary with $M_{\mathrm{NS}}=M_{\mathrm{NS}}^{\min }$ and $M_{\mathrm{BH}}=M_{\mathrm{NS}}^{\max }$ (red line). All GW events with chirp mass below $M_{\mathrm{c}, \mathrm{min}}^{\text {gapNS }}$ are NSNS mergers. Similarly, $M_{\mathrm{c}, \max }^{\mathrm{NSNS}}=1.792 \mathrm{M}_{\odot}$ is the chirp mass corresponding to a NSNS binary with both NSs having the maximum allowed mass (blue line). Events with chirp mass above $M_{\mathrm{c}, \max }^{\mathrm{NSNS}}$ cannot be produced by a NSNS merger. Events with chirp mass between $M_{\mathrm{c}, \min }^{\text {gapN }}$ and $M_{\mathrm{c}, \max }^{\mathrm{NSNS}}$ can be either NSNS or gap $+\mathrm{NS}$ mergers (green-orange lines), i.e. they are "am- 
biguous".

\section{COMPUTATION OF EJECTA PROPERTIES FROM BHNS AND NSNS MERGERS}

During the final phase of a NSNS merger, tidal forces lead to a partial disruption of the stars, producing an outflow of neutron-rich material. When the crusts of the two NS impact each other, compression, shock heating and potentially neutrino ablation cause an additional outflow (Hotokezaka et al. 2013; Bauswein et al. 2013; Radice et al. 2016; Dietrich et al. 2017; Beloborodov et al. 2018). The released NS material can be divided into two components: the dynamical ejecta, gravitationally unbound, that leave the merger region, and a bound component, which forms an accretion disc around the merger remnant. On longer timescales, other outflows originate from the disc: faster ejecta produced by magnetic pressure and neutrino-matter interaction during the initial neutrinocooling-dominated accretion phase (we call these "wind ejecta"), and slower but more massive ejecta produced by viscous processes in the disc, especially during the advection-dominated phase (Dessart et al. 2009; Metzger \& Fernández 2014; Perego et al. 2014; Just et al. 2015; Siegel \& Metzger 2017 - we call these "secular ejecta"). Substantial differences in the ejecta properties arise depending on the post-merger scenario (see i.e. Kawaguchi et al. 2019).

In order to calculate dynamical ejecta and disc mass from a NSNS merger we adopt the fitting formulae reported in Radice et al. (2018), which are calibrated on a large suite of high-resolution GRHD simulations ${ }^{7}$. Both quantities depend on the NS masses and tidal deformabilities. We also adopt their formula for the dynamical ejecta mass-weighted average asymptotic velocity $v_{\text {dyn }}$.

The NS tidal disruption can occur also in BHNS mergers. If the NS is disrupted outside the innermost stable circular orbit, then the released material remains outside the $\mathrm{BH}$ in the form of a crescent (e.g. Kawaguchi et al. 2016), otherwise the NS plunges directly onto the BH. We adopt the fitting formula from Foucart et al. (2018) to calculate the total mass remaining outside the $\mathrm{BH}, M_{\text {out }}$. This quantity depends on the BH mass and spin, and on the NS mass, tidal deformability and baryonic mass $M_{\mathrm{NS}}^{\mathrm{b}}$. We adopt the formulae in Kawaguchi et al. (2016) to calculate the dynamical ejecta mass and average velocity $v_{\mathrm{dyn}}$ in this case. $M_{\mathrm{dyn}}$ depends on the $\mathrm{BH}$ mass and spin, the NS mass, baryonic mass and compactness $C_{\mathrm{NS}}$, and on the angle $\iota_{\text {tilt }}$ between the binary total angular momentum and the $\mathrm{BH}$ spin. We assume $\iota_{\text {tilt }}=0$. $v_{\text {dyn }}$ depends only on the mass ratio $q=M_{\mathrm{BH}} / M_{\mathrm{NS}}$. We proceed as in Barbieri et al. (2019b) to calculate $C_{\mathrm{NS}}$ from $\Lambda_{\mathrm{NS}}$ and $M_{\mathrm{NS}}^{\mathrm{b}}$ from $M_{\mathrm{NS}}$ and $C_{\mathrm{NS}}$. We then calculate the disc mass as $M_{\text {disc }}=\max \left[M_{\text {out }}-M_{\text {dyn }}, 0\right]$. As in Barbieri et al. (2019b) we assume that $M_{\text {dyn }}$ cannot exceed $30 \% M_{\text {out }}$, considering recent BHNS simulations presented in Foucart et al. (2019).

\footnotetext{
7 We note that Kiuchi et al. (2019) showed that the predictions from these formulae might underestimate the produced disc mass in binaries with large mass ratios. However they consider the case with $M_{\mathrm{NS}, 1}=1.55 \mathrm{M}_{\odot}$ and $M_{\mathrm{NS}, 2}=1.2 \mathrm{M}_{\odot}$, thus lowmass/largely deformable NSs. Instead, as can be seen in Fig. 2, we consider systems with $M_{\mathrm{NS}, 1}>1.65 \mathrm{M}_{\odot}$ and $M_{\mathrm{NS}, 2}>1.35 \mathrm{M}_{\odot}$ Therefore the NSs in our systems are less deformable and we expect that the underestimation reported in Kiuchi et al. (2019) is less significant.
}
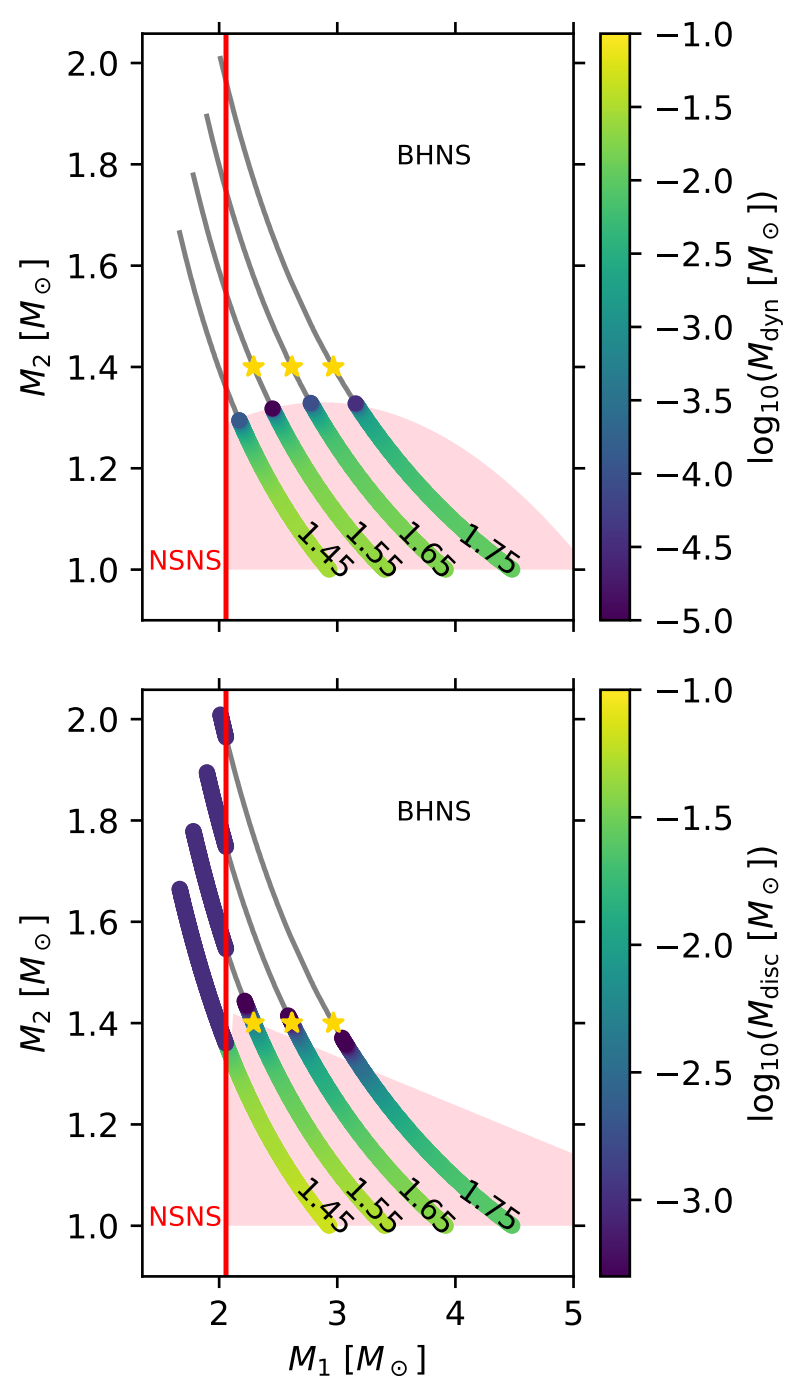

Figure 2. Dynamical ejecta (top panel) and accretion disc (bottom panel) masses for different values of the binary chirp mass $M_{\mathrm{c}}$. We assume the SFHo $\operatorname{EoS}\left(M_{\mathrm{NS}}^{\max }=2.058 \mathrm{M}_{\odot}\right)$ and non-spinning BHs $\left(\chi_{\mathrm{BH}}=0\right)$. The vertical red line separates NSNS configurations (left) and BHNS ones (right). Each line corresponds to a $M_{\mathrm{c}}$ (reported on it). The pink shadowed area is the region where differences of the ejecta mass for the BHNS and NSNS cases are larger than systematic errors. The yellow stars indicate the BHNS systems with the NS having a representative" mass of $1.4 \mathrm{M}_{\odot}$.

In what follows, we conservatively assume the $\mathrm{BH}$ to be non-spinning $\left(\chi_{\mathrm{BH}}=0\right)$, corresponding to the worst condition for ejecta production ${ }^{8}$.

\section{EJECTA PROPERTIES FOR "AMBIGUOUS" CHIRP} MASSES

In Fig. 2 we show the dynamical ejecta and disc masses on the $\left(M_{1}, M_{2}\right)$ plane along lines of constant $M_{\mathrm{c}}$. We limit the $\mathrm{y}$ axis to $M_{\mathrm{NS}}^{\max }$ as we focus on systems that contain at least one NS. It is apparent that NSNS configurations compatible with "ambiguous" chirp masses do

8 among the co-rotating configurations. Indeed the counterrotating cases $\left(\chi_{\mathrm{BH}}<0\right)$ are the worst conditions in absolute, more often leading to a direct plunge. However counter-rotating configurations are not expected for field binaries but for the dynamically formed ones, that represent a negligible contribution to the merger rate (Ye et al. 2019). 


$$
\begin{gathered}
1.46 \mathrm{M}_{\odot} \mathrm{NS}-1.27 \mathrm{M}_{\odot} \mathrm{NS} \\
(\mathrm{GW170817} \text {-like }), \mathrm{M}_{\text {chirp }}=1.18 \mathrm{M}_{\odot}
\end{gathered}
$$

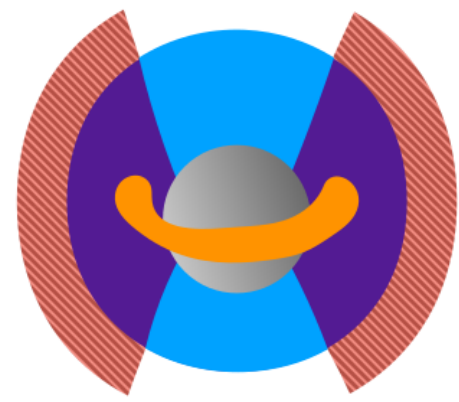

Dynamical Ejecta: $\sim 10^{-3} \mathrm{M}_{\odot}$ Accretion Disc: $\sim 3 \times 10^{-2} \mathrm{M} \odot$ Wind Ejecta: $\sim 3 \times 10^{-3} \mathrm{M}_{\odot}$ Secular Ejecta: $\sim 10^{-2} \mathrm{M} \odot$
II)

$$
\begin{gathered}
2 \mathrm{M}_{\odot} \mathrm{NS}-1.6 \mathrm{M}_{\odot} \mathrm{NS} \\
\mathrm{M}_{\text {chirp }}=1.55 \mathrm{M}_{\odot}
\end{gathered}
$$

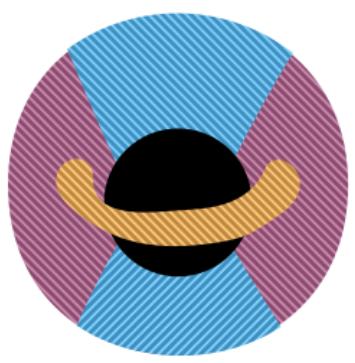

Dynamical Ejecta: Absent Accretion Disc: $\sim 10^{-3} \mathrm{M} \odot$ Wind Ejecta: $\sim 3 \times 10^{-5} \mathrm{M}_{\odot}$ Secular Ejecta: $\sim \mathbf{1 0}^{-4} \mathrm{M}_{\odot}$
III)

$$
\begin{gathered}
3 \mathrm{M}_{\odot} \mathrm{BH}-1.1 \mathrm{M}_{\odot} \mathrm{NS} \\
\mathrm{M}_{\text {chirp }}=1.55 \mathrm{M}_{\odot}
\end{gathered}
$$

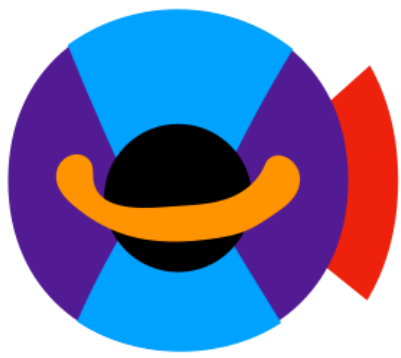

Dynamical Ejecta: $\sim 10^{-2} \mathrm{M}_{\odot}$ Accretion Disc: $\sim 3 \times 10^{-2} \mathrm{M}_{\odot}$ Wind Ejecta: $\sim 3 \times 10^{-4} \mathrm{M}_{\odot}$ Secular Ejecta: $\sim 10^{-2} \mathrm{M}_{\odot}$

Figure 3. Cartoon of the ejecta and disc produced in different systems: (I) a NSNS merger with $\sim 1.46 \mathrm{M}_{\odot}$ and $\sim 1.27 \mathrm{M}_{\odot}$ stars, close to the masses in GW170817; (II) a NSNS merger with two massive stars of $\sim 2 \mathrm{M}_{\odot}$ and $\sim 1.6 \mathrm{M}_{\odot} ;$ and (III) a BHNS merger with a light $\mathrm{BH}$ of $\sim 3 \mathrm{M}_{\odot}$ and a NS of $\sim 1.1 \mathrm{M}_{\odot}$. NSNS and BHNS configurations II and III correspond to the same "ambiguous" chirp mass $M_{\mathrm{c}}=1.55 \mathrm{M}_{\odot}$. Red, orange, light blue and purple represent dynamical ejecta, accretion disc, wind ejecta and secular ejecta, respectively. Filled areas correspond to massive components, while hatched areas correspond to low mass components.

not produce dynamical ejecta (upper panel of Fig. 2). In this parameter region, the fits from Radice et al. (2018) predict the absence of this kind of ejecta, due to the prompt collapse of the remnant to a BH. Conversely, BHNS configurations can more easily produce dynamical ejecta. These systems have small mass ratio $q<5$ and low-mass (large $\Lambda_{\mathrm{NS}}$ ) NSs, which is the optimal condition to produce massive ejecta in BHNS mergers (as shown in Barbieri et al. 2019b,a). The same arguments hold for disc masses (bottom panel in Fig. 2). Note that the value of $M_{\text {disc }}$ predicted by the fitting formula for NSNS systems in the considered range is set by the lower limit indicated in Radice et al. (2018), which is $M_{\text {disc }}=10^{-3}$. For BHNS configurations, instead, discs with masses up to $\sim 7 \times 10^{-2} \mathrm{M}_{\odot}$ are produced.

It is important to note that when the differences of the ejecta mass for the BHNS and NSNS cases are substantial, they are larger than the systematic errors. The uncertainties on the fitting formulae for $\mathrm{NSNS}$ are $\Delta M_{\mathrm{dyn}}^{\mathrm{NSNS}}=0.5 M_{\mathrm{dyn}}+5 \times 10^{-5} \mathrm{M}_{\odot}$ and $\Delta M_{\text {disc }}^{\mathrm{NSNS}}=0.5 M_{\text {disc }}+5 \times 10^{-4} \mathrm{M}_{\odot}$ (Radice et al. 2018). The uncertainties on the fitting formulae for BHNS are $\Delta M_{\mathrm{dyn}}^{\mathrm{BHNS}}=0.2 M_{\mathrm{dyn}}$ (Kawaguchi et al. 2016) and $\Delta M_{\text {out }}=0.1 M_{\text {out }}$ (Foucart et al. 2018). Therefore, being $M_{\text {disc }}^{\mathrm{BHNS}}=M_{\mathrm{out}}-M_{\mathrm{dyn}}^{\mathrm{BHNS}}$, we assume its uncertainty to be $\Delta M_{\text {disc }}^{\mathrm{BHNS}}=\sqrt{\left(\Delta M_{\mathrm{out}}\right)^{2}+\left(\Delta M_{\mathrm{dyn}}^{\mathrm{BHNS}}\right)^{2}}$. We define $\sigma_{\mathrm{dyn}}=\sqrt{\left(\Delta M_{\mathrm{dyn}}^{\mathrm{NSNS}}\right)^{2}+\left(\Delta M_{\mathrm{dyn}}^{\mathrm{BHNS}}\right)^{2}}$ and $\sigma_{\mathrm{disc}}=$ $\sqrt{\left(\Delta M_{\text {disc }}^{\mathrm{NSNS}}\right)^{2}+\left(\Delta M_{\mathrm{disc}}^{\mathrm{BHNS}}\right)^{2}}$. We indicate as pink shadowed area in Fig. 2 the regions where the differences in the mass of dynamical ejecta and disc for the BHNS and NSNS cases are greater than or equal to $\sigma_{\text {dyn }}$ and $\sigma_{\text {disc }}$, respectively. In these regions the ejecta mass differences are larger than the systematic errors.

Figure 3 summarizes the differences between two representative NSNS and BHNS systems with "ambiguous" chirp masses (cases II and III in the Figure), and also a "GW170817-like" NSNS case, for comparison. For the latter we consider a NSNS system with masses $1.46 \mathrm{M}_{\odot}$ and $1.27 \mathrm{M}_{\odot}$.

Merger (I) produces relatively low-mass dynamical ejecta at all latitudes, with a preferentially equatorial angular distribution $\propto \sin ^{2} \theta$, where $\theta$ is the polar angle (Perego et al. 2017). The accretion disc is massive and $\sim 20 \%$ of its mass is unbound in the form of secular ejecta, with a similar angular distribution as for the dynamical ones, while $\sim 5 \%$ of its mass goes into the wind ejecta, mostly confined in the polar region $(\theta<\pi / 3 \mathrm{rad}$ - Perego et al. 2017). After the merger, an intermediate state with a hyper-massive NS could exist before collapsing to a BH (gray central object represented in Fig. 3-I). The strong neutrino winds produced in this state interact with the ejecta, increasing the electron fraction $Y_{\mathrm{e}}$ or, equivalently, lowering the opacity.

We consider a system with $2 \mathrm{M}_{\odot}$ and $1.6 \mathrm{M}_{\odot}$ stars (II) as our representative NSNS merger in the "ambiguous" chirp mass range. As explained above, in this case we expect no dynamical ejecta and a low-mass accretion disc, resulting in low-mass wind and secular ejecta. The merger remnant collapses promptly to a $\mathrm{BH}$. The absence of an intermediate hyper-massive NS state implies little neutrino wind, giving a low $Y_{\mathrm{e}}$ in the ejecta (Kawaguchi et al. 2019).

Finally, as BHNS merger in the "ambiguous" chirp mass range we consider a system with $M_{\mathrm{BH}}=3 \mathrm{M}_{\odot}$ and $M_{\mathrm{NS}}=1.1 \mathrm{M}_{\odot}$ (III). In BHNS mergers, the dynamical ejecta have a crescent-like shape, extending into half of the equatorial plane and limited to the region with $\theta<0.3 \mathrm{rad}$ (Kawaguchi et al. 2016). In the considered system, the dynamical ejecta and accretion disc are massive. Due to the lack of a neutrino wind, the fraction of accretion disc flowing into wind ejecta is lower than in the NSNS case (we assume $\sim 1 \%$ ). The disc fraction that goes into secular ejecta is the same as in the NSNS case. As a consequence, the secular ejecta are massive, while the wind ejecta have low mass. The ejecta $Y_{\mathrm{e}}$ is 
lower than the NSNS case.

Therefore, being the ejecta properties substantially different for the NSNS and BHNS cases in the "ambiguous" chirp mass range, we expect the kilonova light curves to present important differences as well.

\section{KILONOVA MODEL}

The neutron-rich material ejected in NSNS and BHNS mergers is an ideal site for $r$-process nucleosynthesis, which produces the heaviest elements in the Universe (Lattimer \& Schramm 1974; Eichler et al. 1989; Korobkin et al. 2012; Wanajo et al. 2014). The synthesized nuclei are unstable and they decay radioactively, powering the kilonova emission (Li \& Paczyński 1998; Metzger et al. 2010; Kasen et al. 2013).

We compute the kilonova light curves using the composite semi-analytical model presented in Barbieri et al. (2019b,a) (in part based on Perego et al. 2017; Martin et al. 2015; Grossman et al. 2014). For the NSNS cases we assume the model parameters (ejecta geometry, opacity and the fractions of $M_{\text {disc }}$ that go into wind and secular ejecta) as in Perego et al. (2017). The model has been tested on GW170817: using the parameters inferred for this event (Perego et al. 2017; Abbott et al. 2017), we obtain multi-wavelength light curves consistent with the observed ones (Villar et al. 2017, paper in preparation). For BHNS systems we assume the same model parameters as in Barbieri et al. (2019b,a) (based on Kawaguchi et al. 2016; Fernández et al. 2017; Just et al. 2015).

The kilonova light curves are highly degenerate with respect to binary parameters. Thus, it is impossible to infer the system properties from the kilonova light curve alone. This degeneracy can be (at least partially) broken using information from GW analysis. In particular, the measurement of the binary chirp mass reduces the number of parameters by one. Leaving i.e. $M_{1}$ as a free parameter, $M_{2}$ is constrained by the measured $M_{\mathrm{c}}$.

\section{KILONOVAE FOR "AMBIGUOUS" CHIRP MASSES}

In Fig. 4 we show the envelope of the kilonova light curves expected from NSNS and BHNS mergers, for four selected values of the chirp mass. We consider emission in the $\mathrm{g}(509 \mathrm{~nm})$ and $\mathrm{K}(2143 \mathrm{~nm})$ bands and the figure shows the absolute magnitude as a function of time. For all the "ambiguous" chirp masses the fitting formulae from Radice et al. (2018) in NSNS mergers predict no dynamical ejecta and a minimum allowed disc mass $M_{\text {disc }}=10^{-3} \mathrm{M}_{\odot}$. Thus we have a single light curve for NSNS mergers, and we can expect that these events would not produce kilonovae brighter than shown in Fig. 4.

For BHNS mergers there exists a range of light curves for each $M_{\mathrm{c}}$, arising from the different combinations of the component masses, producing different ejecta properties. For $M_{\mathrm{c}}=1.45 \mathrm{M}_{\odot}$ (panels 1a-1b) all kilonovae from BHNS mergers are much brighter at every time than that from NSNS mergers. Therefore a single observation in one of these bands would allow in principle to distinguish the nature of the merging system.

At higher values of the chirp mass, there is only a small overlap between the BHNS and NSNS cases, at the bottom of the BHNS envelope. Therefore, except for observed light curves at low absolute magnitudes, it should be always possible to distinguish the nature of the merging system by the sole kilonova brightness. We note that the disentangling of the nature of the binary is optimal when $M_{\mathrm{c}}=1.45 \mathrm{M}_{\odot}$ (panels $\left.1 \mathrm{a}-1 \mathrm{~b}\right)$. In this case, as shown in Fig. 2, the mass interval of the ejecta from BHNS mergers is the narrowest, and this in turn leads to the narrowest spread in the kilonova light curves.

The prediction of BHNS kilonovae as bright or brighter than NSNS ones is presented in Kawaguchi et al. (2019). They find that BHNS kilonovae are brighter in the nearinfrared K-band, due to the smaller electron fraction $Y_{\mathrm{e}}$ in the ejecta owing to the lack of strong neutrino irradiation from the central remnant. In the i band, Kawaguchi et al. (2019) find that NSNS configurations ending with the formation of a supermassive NS leads to brighter kilonovae than the BHNS case. This is due to the strong neutrino emission produced in this case, that increases $Y_{\mathrm{e}}$ in the ejecta. However, in their study they compare sundry BHNS and NSNS configurations not selected on the bases of the chirp mass. By contrast, in our work, we compare BHNS and NSNS mergers at fixed chirp mass. This requirement restricts the NSNS binary configurations to cases producing no dynamical ejecta and very low mass discs. Therefore, whatever the value of $Y_{\mathrm{e}}$ in the ejecta from NSNS merger is, the mass propelled in the merger is so low that almost all the BHNS kilonovae are brighter, at all wavelengths.

For other comparisons between NSNS and BHNS merger outcomes and studies on distinguishing the nature of merging compact binaries see Hinderer et al. (2019); Coughlin \& Dietrich (2019), who considered an unconventional $\mathrm{BH}$ companion with mass of $\sim 1.4 \mathrm{M}_{\odot}$, thus below the maximum NS mass.

As a visual comparison we also show the kilonovae for BHNS binaries having a NS with a representative" mass of $1.4 \mathrm{M}_{\odot}($ aqua/magenta lines $)$. For $M_{\mathrm{c}}=1.45 \mathrm{M}_{\odot}$ such a binary does not exist, while for $M_{\mathrm{c}}=1.75 \mathrm{M}_{\odot}$ it is fated to a direct plunge, thus there is no kilonova.

We remark that the kilonova light curves from BHNS are inferred assuming non-spinning BHs $\left(\chi_{\mathrm{BH}}=0\right)$. As explained in Barbieri et al. (2019b,a), increasing the $\mathrm{BH}$ spin (fixing all the other parameters) leads to more massive ejecta and, consequently, more luminous kilonovae. Therefore, if the BHs have a non-zero spin, our argument would be even stronger. As an example, for $\chi_{\mathrm{BH}} \gtrsim 0.5$ all light curves from BHNS kilonovae would be brighter than those from NSNS binaries in each band and at any time, in this critical range of ambiguous" chirp masses.

\section{CONCLUSION}

The detection of a BHNS coalescence could be the next ground-breaking discovery in multi-messenger astronomy. At the time of writing, there are promising GW candidates detected by the LVC during the observation run O3. The associated detection of an electromagnetic signal from these new GW sources might contribute to our understanding of the physical processes that power the multi-wavelength EM emission (Gompertz et al. 2018; Rossi et al. 2019, and references therein).

From the GW signal, one of the best constrained parameters in low latency is the binary chirp mass, a combination of the masses of the two components. This parameter is currently used to classify the binary, whether the system hosts two NSs, two stellar BHs or a $\mathrm{BH}$ and 

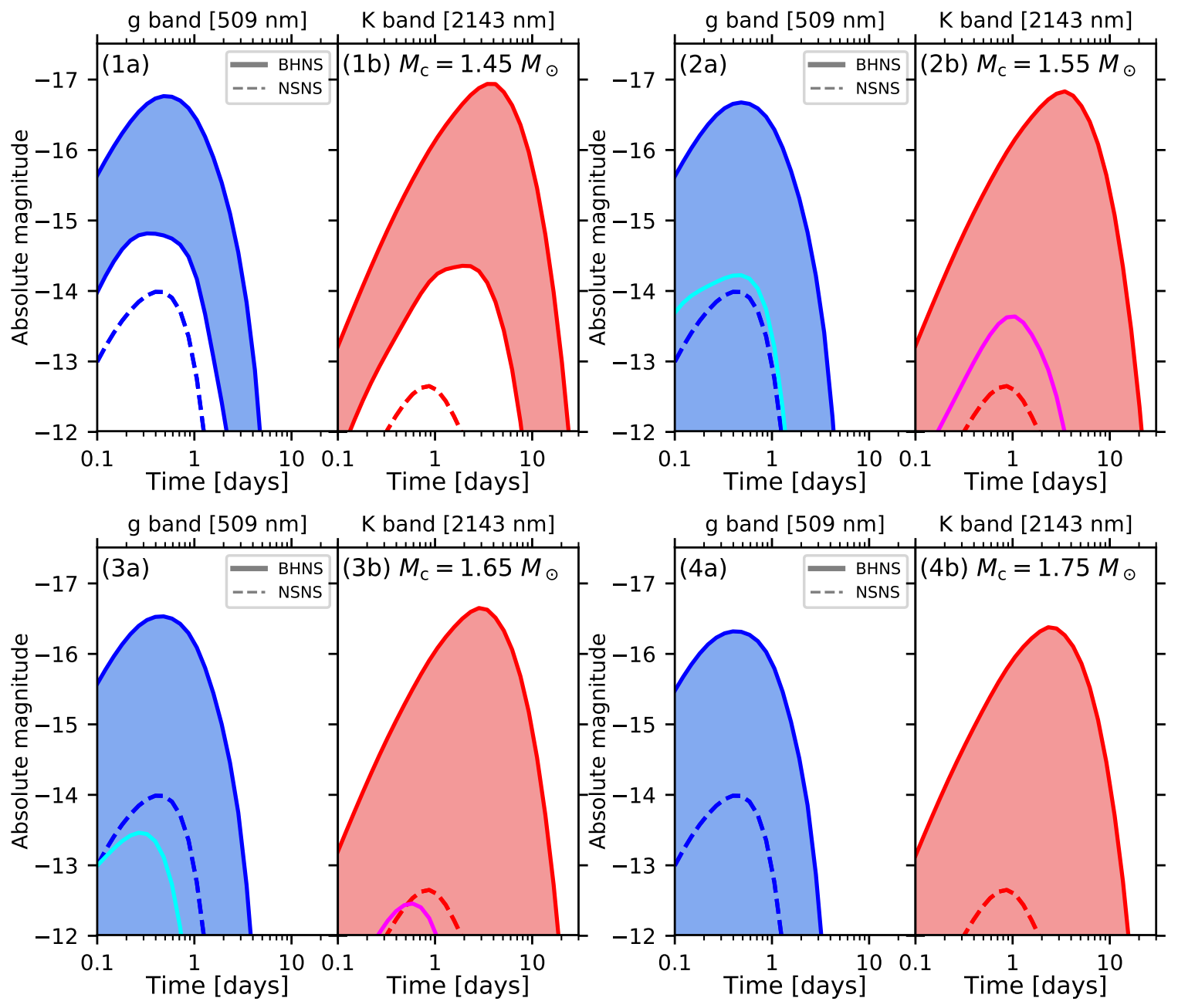

Figure 4. Light curve ranges for kilonovae produced by NSNS (dashed lines) and BHNS (filled areas) binaries with a chirp mass $M_{\mathrm{C}}=$ $1.45 \mathrm{M}_{\odot}(1), 1.55 \mathrm{M}_{\odot}(2), 1.65 \mathrm{M}_{\odot}(3)$ and $1.75 \mathrm{M}_{\odot}(4)$. The a" and b" panels show light curve ranges in the $\mathrm{g}(509 \mathrm{~nm})$ and $\mathrm{K}(2143 \mathrm{~nm})$ band, respectively. Aqua (magenta) lines represent the kilonova in the $\mathrm{g}(\mathrm{K})$ band for the BHNS systems with a NS having a representative mass of $1.4 \mathrm{M}_{\odot}$.

a NS. In the present Letter, we point out that in absence of a "mass gap" between the NS and BH mass distributions (as Thompson et al. 2019 seem to indicate), there exists a range of $M_{\mathrm{c}}$ (as shown in Fig. 1) for which it is not possible to distinguish the nature of the binary on the basis of the chirp mass measurement alone ${ }^{9}$. For the SFHo EoS adopted in this analysis, we find that the values of the chirp mass between $1.233 \mathrm{M}_{\odot}$ and $1.792 \mathrm{M}_{\odot}$ are compatible either with NSNS and BHNS systems.

In this Letter we show that the observation of the kilonova emission from these systems can break the degeneracy in the "ambiguous" chirp mass range, and constrain the nature of the merging system. We find that kilonova emission shows substantial differences in the lu-

9 Offline GW signal analysis could provide more precise information. This would lead to stronger constraints on the component masses that could allow to distinguish the nature of the merging system using GW alone, in some cases. minosity and temporal evolution in NSNS and BHNS systems (see Fig. 4). In particular, the BHNS case is far more luminous than the NSNS case, even when the BH is non-spinning. This happens because in in this "ambiguous" $M_{\mathrm{c}}$ range the NSNS configurations represent the worst cases for ejecta production, while the BHNS configurations represent the best ones. It is important to note that, when the differences of the ejecta mass for the BHNS and NSNS cases are substantial, they are larger than the systematic errors in the modeling. Therefore, observing the kilonova associated with such an event is of fundamental importance to break the degeneracy on the nature of the merging system. Furthermore, if the observed kilonova is compatible with a BHNS merger, this would provide evidence in support of the existence of low-mass BHs, filling the "mass gap".

This work illustrates the potential of multi-messenger observations of compact binary mergers, and the impor- 
tance of an efficient exchange of information between the GW and EM communities (Biscoveanu et al. 2019; Margalit \& Metzger 2019).

We thank F. Zappa and S. Bernuzzi for sharing EoS tables. The authors acknowledge support from INFN, under the Virgo-Prometeo initiative. O. S. and G.G. acknowledges the Italian Ministry for University and Research (MIUR) for funding through project grant 1.05.06.13.

\section{REFERENCES}

Abbott, B. P., Abbott, R., Abbott, T. D., et al. 2017, ApJL, 850, L39

Abbott, B. P., Abbott, R., Abbott, T. D., et al. 2017a, Phys. Rev. Lett., 119, 161101

Abbott, B. P., et al. 2017b, Astrophys. J., 848, L12

Barbieri, C., Salafia, O. S., Perego, A., Colpi, M., \& Ghirlanda, G. 2019a, arXiv e-prints, arXiv:1908.08822

-. 2019b, A\&A, 625, A152

Bauswein, A., Goriely, S., \& Janka, H. T. 2013, ApJ, 773, 78

Belczynski, K., Wiktorowicz, G., Fryer, C. L., Holz, D. E., \& Kalogera, V. 2012, The Astrophysical Journal, 757, 91

Beloborodov, A. M., Lundman, C., \& Levin, Y. 2018, arXiv e-prints, arXiv:1812.11247

Biscoveanu, S., Vitale, S., \& Haster, C.-J. 2019, arXiv e-prints, arXiv:1908.03592

Coughlin, M. W., \& Dietrich, T. 2019, arXiv e-prints, arXiv:1901.06052

Coughlin, M. W., Dietrich, T., Antier, S., et al. 2019, arXiv e-prints, arXiv: 1910.11246

Crisp, H. 2019, Gamma-ray Coordinates Network Circulars, 25749

Cromartie, H. T., Fonseca, E., Ransom, S. M., et al. 2019, Nature Astronomy, 439

D’Avanzo, P., Campana, S., Salafia, O. S., et al. 2018, A\&A, 613, L1

Dessart, L., Ott, C. D., Burrows, A., Rosswog, S., \& Livne, E. 2009, ApJ, 690, 1681

Dietrich, T., Ujevic, M., Tichy, W., Bernuzzi, S., \& Brügmann, B. 2017, Phys. Rev. D, 95, 024029

Dominik, M., Berti, E., O'Shaughnessy, R., et al. 2015, ApJ, 806, 263

Eichler, D., Livio, M., Piran, T., \& Schramm, D. N. 1989, Nature, 340,126

Fernández, R., Foucart, F., Kasen, D., et al. 2017, Classical and Quantum Gravity, 34, 154001

Foucart, F., Duez, M. D., Kidder, L. E., et al. 2019, Phys. Rev. D, 99, 103025

Foucart, F., Hinderer, T., \& Nissanke, S. 2018, ArXiv e-prints, arXiv: 1807.00011

Fryer, C. L., Belczynski, K., Wiktorowicz, G., et al. 2012, ApJ, 749, 91

Ghirlanda, G., Salafia, O. S., Paragi, Z., et al. 2018, arXiv e-prints, arXiv:1808.00469

Gompertz, B. P., Levan, A. J., Tanvir, N. R., et al. 2018, ApJ, 860, 62

Grossman, D., Korobkin, O., Rosswog, S., \& Piran, T. 2014, MNRAS, 439, 757

Hinderer, T., Nissanke, S., Foucart, F., et al. 2019, Phys. Rev. D, 100, 063021

Hotokezaka, K., Kiuchi, K., Kyutoku, K., et al. 2013, Phys. Rev. D, 87, 024001

Just, O., Bauswein, A., Ardevol Pulpillo, R., Goriely, S., \& Janka, H. T. 2015, MNRAS, 448, 541

Kasen, D., Badnell, N. R., \& Barnes, J. 2013, ApJ, 774, 25

Kawaguchi, K., Kyutoku, K., Shibata, M., \& Tanaka, M. 2016, ApJ, 825, 52

Kawaguchi, K., Shibata, M., \& Tanaka, M. 2019, arXiv e-prints, arXiv:1908.05815
Kiuchi, K., Kyutoku, K., Shibata, M., \& Taniguchi, K. 2019, ApJL, 876, L31

Klotz, A. 2019, Gamma-ray Coordinates Network Circulars, 25338 Korobkin, O., Rosswog, S., Arcones, A., \& Winteler, C. 2012, MNRAS, 426, 1940

Lattimer, J. M., \& Prakash, M. 2001, ApJ, 550, 426

Lattimer, J. M., \& Schramm, D. N. 1974, ApJL, 192, L145

Li, L.-X., \& Paczyński, B. 1998, ApJL, 507, L59

Mandel, I., Haster, C.-J., Dominik, M., \& Belczynski, K. 2015, MNRAS, 450, L85

Margalit, B., \& Metzger, B. D. 2019, ApJL, 880, L15

Martin, D., Perego, A., Arcones, A., et al. 2015, ApJ, 813, 2

Metzger, B. D. 2017, Living Reviews in Relativity, 20, 3

Metzger, B. D., \& Fernández, R. 2014, MNRAS, 441, 3444

Metzger, B. D., Martínez-Pinedo, G., Darbha, S., et al. 2010, MNRAS, 406, 2650

Narayan, R., Paczynski, B., \& Piran, T. 1992, ApJL, 395, L83

Özel, F., Psaltis, D., Narayan, R., \& McClintock, J. E. 2010, ApJ, 725, 1918

Paschalidis, V. 2017, Classical and Quantum Gravity, 34, 084002

Perego, A., Radice, D., \& Bernuzzi, S. 2017, ApJ, 850, L37

Perego, A., Rosswog, S., Cabezón, R. M., et al. 2014, MNRAS, 443,3134

Pereyra, M. 2019, Gamma-ray Coordinates Network Circulars, 25737

Radice, D., Galeazzi, F., Lippuner, J., et al. 2016, MNRAS, 460, 3255

Radice, D., Perego, A., Hotokezaka, K., et al. 2018, ApJ, 869, 130

Rossi, A., Stratta, G., Maiorano, E., et al. 2019, arXiv e-prints, arXiv:1901.05792

Ruiz, M., Shapiro, S. L., \& Tsokaros, A. 2018, ArXiv e-prints, arXiv: 1810.08618

Salafia, O. S., Ghirlanda, G., Ascenzi, S., \& Ghisellini, G. 2019, arXiv e-prints, arXiv: 1905.01190

Sari, R., Piran, T., \& Narayan, R. 1998, ApJ, 497, L17

Shapiro, S. L. 2017, Phys. Rev. D, 95, 101303

Shibata, M., \& Taniguchi, K. 2011, Living Reviews in Relativity, 14,6

Siegel, D. M., \& Metzger, B. D. 2017, Phys. Rev. Lett., 119, 231102

Soares-Santos, M. 2019, Gamma-ray Coordinates Network Circulars, 25336

Srivastav, S. 2019, Gamma-ray Coordinates Network Circulars, 25324

Steiner, A. W., Hempel, M., \& Fischer, T. 2013, ApJ, 774, 17

Suwa, Y., Yoshida, T., Shibata, M., Umeda, H., \& Takahashi, K. 2018, MNRAS, 481, 3305

The LIGO Scientific Collaboration, \& the Virgo Collaboration. 2018, arXiv e-prints, arXiv:1811.12907

—. 2019a, Gamma-ray Coordinates Network Circulars, 25324

—. 2019b, Gamma-ray Coordinates Network Circulars, 25695

—. 2019c, Gamma-ray Coordinates Network Circulars, 25829

-. 2019d, Gamma-ray Coordinates Network Circulars, 25871

The LIGO Scientific Collaboration, the Virgo Collaboration, Abbott, B. P., et al. 2018, arXiv e-prints, arXiv:1811.12940

Thompson, T. A., Kochanek, C. S., Stanek, K. Z., et al. 2019, Science, 366, 637

Villar, V. A., Guillochon, J., Berger, E., et al. 2017, ApJL, 851, L21

Wanajo, S., Sekiguchi, Y., Nishimura, N., et al. 2014, ApJL, 789, L39

Ye, C. S., Fong, W.-f., Kremer, K., et al. 2019, arXiv e-prints, arXiv:1910.10740

Zhang, J., Yang, Y., Zhang, C., et al. 2019, MNRAS, 488, 5020 